\title{
An iterative destriping technique for diffuse background polarization data
}

\author{
C. Sbarra ${ }^{1}$, E. Carretti ${ }^{1}$, S. Cortiglioni ${ }^{1}$, M. Zannoni ${ }^{2}$, R. Fabbri $^{3}$, C. Macculi ${ }^{1}$, and M. Tucci ${ }^{4}$ \\ 1 IASF-CNR Bologna, via P. Gobetti 101, 40129 Bologna, Italy \\ e-mail: carretti@bo.iasf.cnr.it; cortiglioni@bo.iasf.cnr.it; macculi@bo.iasf.cnr.it \\ 2 IASF-CNR Milano, via Bassini 15, 20133 Milano, Italy \\ e-mail: zannoni@mi .iasf.cnr.it \\ 3 Dipartimento di Fisica, Università di Firenze, via Sansone 1, 50019 Sesto Fiorentino, Italy \\ e-mail: fabbrir@unifi.it \\ ${ }^{4}$ Instituto de Fisica de Cantabria, Avda. Los Castros s/n, 39005 Santander, Spain \\ e-mail: tucci@ifca.unican.es
}

Received 18 December 2002 / Accepted 13 February 2003

\begin{abstract}
We describe a simple but effective iterative procedure specifically designed to destripe $Q$ and $U$ Stokes parameter data as those collected by the SPOrt experiment onboard the International Space Station (ISS). The method is general enough to be useful for other experiments, both in polarization and total intensity. The only requirement for the algorithm to work properly is that the receiver knee frequency must be lower than the signal modulation frequency, corresponding in our case to the ISS orbit period. Detailed performances of the technique are presented in the context of the SPOrt experiment, both in terms of added rms noise and residual correlated noise.
\end{abstract}

Key words. cosmic microwave background - polarization - cosmology: observations - methods: data analysis - methods: numerical

\section{1. introduction}

Low frequency noise is known to affect all radiometers and to induce correlations among successive samples of the measured signal, leading to typical striping effects when producing sky maps. It is characterised by a power-law spectrum $S(f) \propto$ $(1 / f)^{\beta}$, with $\beta$ roughly in the range $1 \div 2.5$ depending on the noise source. The total instrumental noise power spectrum is usually specified in terms of the knee frequency, $f_{\mathrm{k}}$, at which the white and low frequency components of the noise are equal:

$S(f)=\sigma_{0}^{2}\left[1+\left(\frac{f_{\mathrm{k}}}{f}\right)^{\beta}\right]$

where $\sigma_{0}^{2}$ represents the white noise variance per second. As shown by Janssen et al. (1996), when data are taken from spinning spacecrafts, where the spin acts as an ideal switch or chopper, most of the low frequency noise can be removed by software, provided $f_{\mathrm{k}}$ is lower than the satellite spin frequency, $f_{\mathrm{s}}$.

For present and future space experiments primarily dedicated to Cosmic Microwave Background (CMB)

Send offprint requests to: $\mathrm{C}$. Sbarra, e-mail: sbarra@bo.iasf.cnr.it anysotropy measurements, like MAP ${ }^{1}$ and $\mathrm{PLANCK}^{2}$, destriping techniques have already been set up to clean the Time Ordered Data (TOD) from low frequency noise. The impact of different scanning strategies on the quality of final maps has been studied as well (Delabrouille 1998; Maino et al. 1999).

The problem of removing $1 / f$ noise from the data stream is even more important for experiments attempting measurements of CMB polarization (CMBP), where even a tiny amount of residual correlated noise, at a level negligible for investigations of CMB temperature anysotropy $\left(\Delta T / T \simeq 10^{-5}\right)$, might distort the signal characteristics: in the most favourable case the expected polarization level is only in the order of $10 \%$ of the temperature fluctuations. The first algorithm specifically designed for destriping polarization data has been proposed by Revenu et al. (2000), who extended to polarization data ideas previously studied to destripe PLANCK anisotropy data (Delabrouille 1998).

The SPOrt ${ }^{3}$ experiment onboard the ISS (Cortiglioni et al. 2002; Carretti et al. 2003) is equipped with 4 correlation polarimeters directly measuring the $Q$ and $U$ Stokes parameters in the 22-90 GHz frequency range, with $F W H M=7^{\circ}$.

\footnotetext{
1 MAP homepage: http://map.gsfc.nasa.gov

2 PLANCK homepage: http://astro.estec.esa.nl/Planck

${ }^{3}$ SPOrt homepage: http://sport.bo.iasf.cnr.it
} 

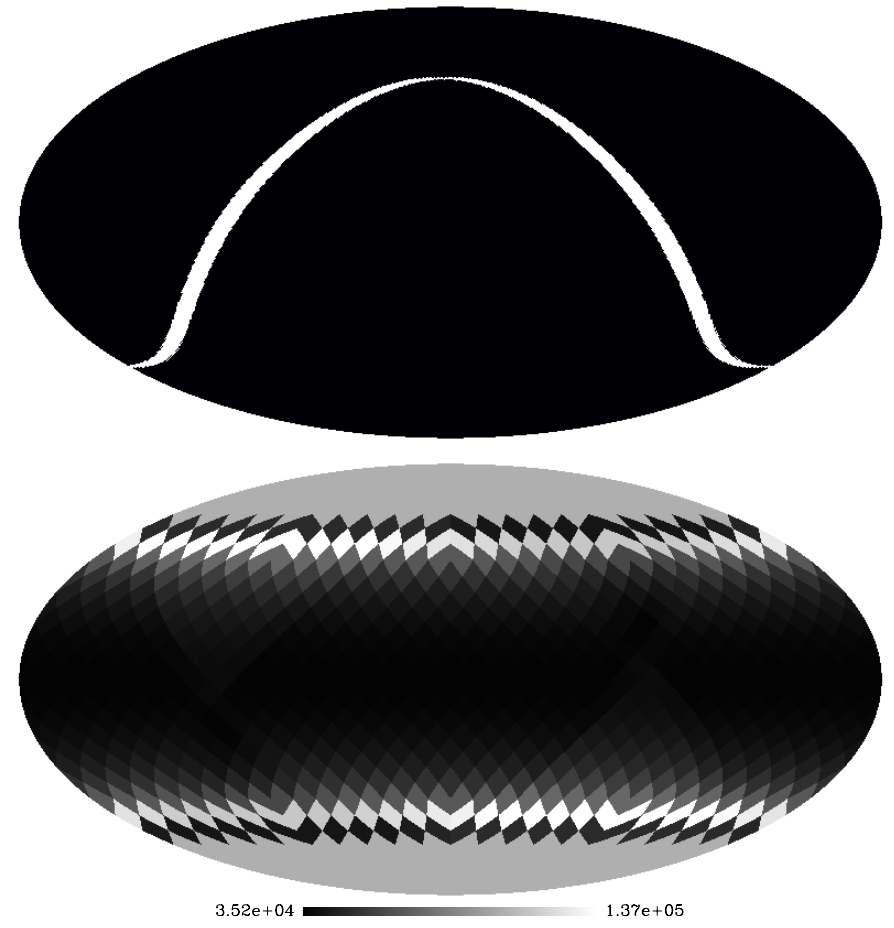

Fig. 1. Top: the sky, in Celestial coordinates, as scanned by SPOrt in few orbits. Bottom: pixel observing time (seconds) for 388 days of data taking. The pixel size is about $7^{\circ}$ (HEALPix parameter $N$ side $=8$ ).

Besides providing polarization maps of Galactic synchrotron, SPOrt will try a first detection of CMBP on large angular scales $(\ell \leq 25)$, where the CMB E-mode power spectrum is particularly sensitive to the value of the optical depth of the reionized medium (Seljak 1997; Zaldarriaga et al. 1997). Such information cannot be extracted from CMB temperature anisotropy data, nor from CMBP data taken by balloon and ground-based experiments looking at small sky patches. This makes CMBP measurements on large angular scale of the utmost importance.

The algorithm presented here is particularly suited to SPOrt, acting directely on $Q$ and $U$ Stokes parameter data.

The SPOrt radiometers are based on InP HEMT amplifiers, for which the low frequency noise is known to have a $1 / f$-like spectrum $(\beta \simeq 1)$ dominated by transistor gain fluctuations (Wollack 1995), with knee frequencies in the range $100-1000 \mathrm{~Hz}$. However, when using correlation techniques, the knee frequency is reduced by a factor $\left(T_{\text {offset }} / T_{\text {sys }}\right)^{2}$, with $T_{\text {offset }}$ and $T_{\text {sys }}$ the instrumental offset and the system noise temperature, respectively (Wollack \& Pospiesalski 1998; Carretti et al. 2001). The present state of the instrument already guarantees a value $f_{\mathrm{k}}<2.5 \times 10^{-5} \mathrm{~Hz}$ (Carretti et al. 2003), close to the goal knee frequency of one tenth of the ISS orbit frequency, i.e. $f_{\mathrm{k}}^{\text {goal }}=1.8 \times 10^{-5} \mathrm{~Hz}$.

The SPOrt scanning strategy is bound to the ISS motion. The ISS orbit is tilted by $51.6^{\circ}$ with respect to the Celestial equator and is characterized by a frequency $f_{\mathrm{o}}=1.8 \times 10^{-4} \mathrm{~Hz}$. The SPOrt antennae, looking at the zenit, will cover about $80 \%$ of the sky under precessing circles intersecting each other. The nominal sampling rate is $1 \mathrm{~Hz}$, and a sky map will be provided every 70 days in a mission lasting at least 18 months. Further details about the SPOrt sky coverage are shown in Fig. 1.

The iterative algorithm described in this paper has been specifically designed to destripe $Q$ and $U$ Stokes parameter data. It is general enough to be valid for any experiment where data are taken by scanning the sky at a frequency higher than the receiver knee frequency, and where different scans intersect each other in a sufficient number of points. Measurements of scalar quantities are easily handled.

The description of the algorithm is provided in Sect. 2, whereas Sect. 3 presents various tests of its performances and Sect. 4 summarises our conclusions.

\section{Destriping method}

The only assumptions our algorithm relies upon is that the radiometer is stable during the signal modulation period (the time needed to complete one orbit in case of SPOrt), so that the instrumental offset does not change significantly during this time, and there is enough overlap between different orbits. In such a case the noise can be split in two parts: for time scales shorter than the orbit period (high frequencies) it is essentially white, whereas for longer timescales (low frequencies) it also contains the $1 / f$ component, which we model as a different constant offset for each orbit. A simple iterative procedure is then applied to remove these offsets from the TOD before map-making. The map-making itself consists in averaging the cleaned TOD corresponding to the same sky pixel after rotating them into a fixed reference frame. Parallel transport to the center of each pixel is also implemented, as described by Bruscoli et al. (2002), before averaging, and can optionally be switched on, though it is not strictly necessary for the portion of sky covered by SPOrt. It would be mandatory if polar caps were included in the accessible region. The time needed to simulate 1 year of SPOrt data taking with the nominal sampling rate of $1 \mathrm{~Hz}$, destripe the TOD and produce $Q$ and $U$ clean maps is about $10 \mathrm{~min}$ on a $1.3 \mathrm{GHz}$ Pentium III equipped with a 4 GB RAM when using 10 destriping cycles, and increases by 2.5 min for each additional bunch of 10 iteration cycles.

\subsection{Formalism}

Each datum consists of a $(\tilde{Q}, \tilde{U})$ pair as measured in the polarimeter reference frame: the same sky point, when seen from different orbits, shows different $(\tilde{Q}, \tilde{U})$ values even in absence of noise. The standard longitude-latitude fixed reference frame (Berkhuijsen 1975) is used when considering the real sky emission, which is projected into an $N$-pixel map. Our $M$ TOD, collected during $R$ orbits about the Earth, are used to build an $M$-vector $\boldsymbol{Y}$ of measured $(\tilde{Q}, \tilde{U})$ pairs:

$\boldsymbol{Y}=\boldsymbol{A} \cdot \boldsymbol{X}+\boldsymbol{B} \cdot \mathrm{OFF}+\boldsymbol{N}$.

$\boldsymbol{X}$ is an $N$-vector whose elements are the $(Q, U)$ pairs of the true sky emission; $\boldsymbol{A}$ is an $M \times N$ pointing matrix connecting sky pixels to observations (Wright 1996; Tegmark 1997a): its elements are $2 \times 2$ matrices rotating the Stokes parameters from the fixed to the polarimeter reference frame; $\boldsymbol{O F F}$ is an $R$-vector containing a pair of radiometric offsets for each orbit; $\boldsymbol{B}$ is an 
$M \times R$ pointing matrix connecting orbits to observations, and $N$ is an $M$-vector containing pairs of white noise. In more details, the elements of the above mentioned vectors and matrices are:

$Y_{t}=\left(\begin{array}{c}\tilde{Q}_{t} \\ \tilde{U}_{t}\end{array}\right)$

$A_{t p}= \begin{cases}\mathcal{R}\left(\alpha_{t}\right)=\left(\begin{array}{cc}\cos \left(2 \alpha_{t}\right) & \sin \left(2 \alpha_{t}\right) \\ -\sin \left(2 \alpha_{t}\right) & \cos \left(2 \alpha_{t}\right)\end{array}\right) & \begin{array}{l}\text { if pixel } p \\ \text { is observed } \\ \text { at time } t\end{array} \\ \left(\begin{array}{ll}0 & 0 \\ 0 & 0\end{array}\right) & \text { otherwise }\end{cases}$

where $\alpha_{t}$ is the angle between the polarimeter and the fixed reference frames at time $t$.

$X_{p}=\left(\begin{array}{c}Q_{p} \\ U_{p}\end{array}\right)$

$B_{t o}= \begin{cases}\left(\begin{array}{ll}1 & 0 \\ 0 & 1\end{array}\right) & \text { if } o \text { is the orbit run at time } t \\ \left(\begin{array}{ll}0 & 0 \\ 0 & 0\end{array}\right) & \text { otherwise }\end{cases}$

$O f f_{o}=\left(\begin{array}{c}O f f_{o}^{\tilde{Q}} \\ O f f_{o}^{\tilde{U}}\end{array}\right)$

$N_{t}=\left(\begin{array}{c}N_{t}^{\tilde{Q}} \\ N_{t}^{\tilde{U}}\end{array}\right)$.

\subsection{Iteration cycle}

If we knew the sky emission vector $\boldsymbol{X}$ we could use it to obtain the offset vector $\boldsymbol{O F F}$ by inverting Eq. (2). Of course, estimating the noise offsets would not be worthwhile if we already knew the true sky emission. However, to start we can guess a 0-order sky emission $\mathbf{X}^{(\mathbf{0})}$, the most neutral choice being a null vector; then obtain a 0 -order offset estimate, and subtract it from our measurements to get a 0-order cleaned TOD; from this compute $\mathbf{X}^{(\mathbf{1})}$, the 1st-order estimate of the real sky emission, and iterate the cycle.

Starting from Eq. (2) and following Tegmark (1997b), the best estimators for the offset vector and the signal matrix can be written:

$$
\begin{aligned}
\boldsymbol{O F F} & =\boldsymbol{W}_{\mathrm{obs}}(\boldsymbol{Y}-\boldsymbol{A} \cdot \boldsymbol{X}) \\
\boldsymbol{X} & =\boldsymbol{W}_{\mathrm{pix}}(\boldsymbol{Y}-\boldsymbol{B} \cdot \boldsymbol{O F F})
\end{aligned}
$$

where, in case of uniform white-noise variance, as expected for the SPOrt receivers,

$$
\begin{aligned}
& \boldsymbol{W}_{\text {obs }}=\boldsymbol{N}_{\text {obs }}^{-1} \cdot \boldsymbol{B}^{\mathrm{T}} \\
& \boldsymbol{W}_{\text {pix }}=\boldsymbol{N}_{\text {pix }}^{-1} \cdot \boldsymbol{A}^{\mathrm{T}}
\end{aligned}
$$

with $\boldsymbol{N}_{\text {obs }}=B^{\mathrm{T}} \boldsymbol{B}$ and $\boldsymbol{N}_{\text {pix }}=\boldsymbol{A}^{\mathrm{T}} \boldsymbol{A}$ diagonal matrices containing the number of observations in each orbit and in each pixel, respectively. In such a case Eq. (10) simply corresponds to rotating into the fixed reference frame the offset-subtracted observations and averaging those corresponding to the same sky pixel. When starting from a null guess map, i.e. $\boldsymbol{X}^{(0)}=0$, the first offset estimate is a simple average of the measurements taken over each orbit.

In the more general case of non-uniform white noise, i.e. when the noise covariance matrix $C=\left\langle\boldsymbol{N} N^{\mathrm{T}}\right\rangle$ has unequal diagonal elements, the matrices $\boldsymbol{W}_{\text {obs }}$ and $\boldsymbol{W}_{\text {pix }}$ become (Tegmark 1997b)

$$
\begin{aligned}
& \boldsymbol{W}_{\text {obs }}=\left(\boldsymbol{B}^{\mathrm{T}} \boldsymbol{C}^{-1} \boldsymbol{B}\right)^{-1} \boldsymbol{B}^{\mathrm{T}} \boldsymbol{C}^{-1} \\
& \boldsymbol{W}_{\text {pix }}=\left(\boldsymbol{A}^{\mathrm{T}} \boldsymbol{C}^{-1} \boldsymbol{A}\right)^{-1} \boldsymbol{A}^{\mathrm{T}} \boldsymbol{C}^{-1}
\end{aligned}
$$

meaning that the simple averages mentioned above should be replaced with noise-weighted averages.

In any case the first offset estimate writes:

$\boldsymbol{O F} \boldsymbol{F}^{(0)}=\boldsymbol{W}_{\text {obs }}^{-1} \boldsymbol{Y}$.

The 0-order cleaned TOD are:

$\boldsymbol{Y}^{(0)}=\boldsymbol{Y}-\boldsymbol{B} \cdot \boldsymbol{O} \boldsymbol{F} \boldsymbol{F}^{(0)}$

and the 1st-order estimate of the true sky emission is

$\boldsymbol{X}^{(1)}=\boldsymbol{W}_{\mathrm{pix}} \cdot \boldsymbol{Y}^{(0)}$.

The $i$ th cycle writes:

$$
\begin{aligned}
\boldsymbol{O F F}^{(i)} & =\boldsymbol{W}_{\mathrm{obs}}\left(\boldsymbol{Y}-\boldsymbol{A} \cdot \boldsymbol{X}^{(i)}\right) \\
\boldsymbol{X}^{(i+1)} & =\boldsymbol{W}_{\mathrm{pix}}\left(\boldsymbol{Y}-\boldsymbol{B} \cdot \boldsymbol{O} \boldsymbol{F} \boldsymbol{F}^{(i)}\right)
\end{aligned}
$$

and must be iterated until the difference between the sky emission estimates of order $i$ and $i+1$ is negligible when compared to the white noise level.

The proof that the procedure is effective has been obtained numerically, as described in detail in the next section. Destriping measurements of total intensity (or, in general, any other scalar quantity) can be simply performed by replacing pairs with scalars in the previously defined vectors, and rotation matrices with scalar 1 . The average sky signal is lost when destriping maps of scalar quantities. However, for maps of polarization data like $Q$ and $U$ it can be kept provided the polarimeter reference frame rotates about the fixed frame while running along each orbit, as in the case of SPOrt. This is a nice feature, especially when measuring foreground contributions.

\section{Simulations and tests}

We test the method with numerical simulations of the data stream expected from about one year of SPOrt data taking.

As real sky emission we consider both CMB and synchrotron radiation, both convolved with a $7^{\circ}$ Gaussian beam. Other foregrounds are expected to be less important at the frequencies and angular scales covered by SPOrt (Tegmark et al. 2000; Lazarian \& Prunet 2002) and are not included.

CMB emission is generated by the $\mathrm{CMBFAST}^{4}$ package according to a $\Lambda$ CDM cosmological model with $\Omega_{\mathrm{m}}=0.3$,

\footnotetext{
4 http://www.physics.nyu.edu/matiasz/CMBFAST/ cmbfast.html
} 


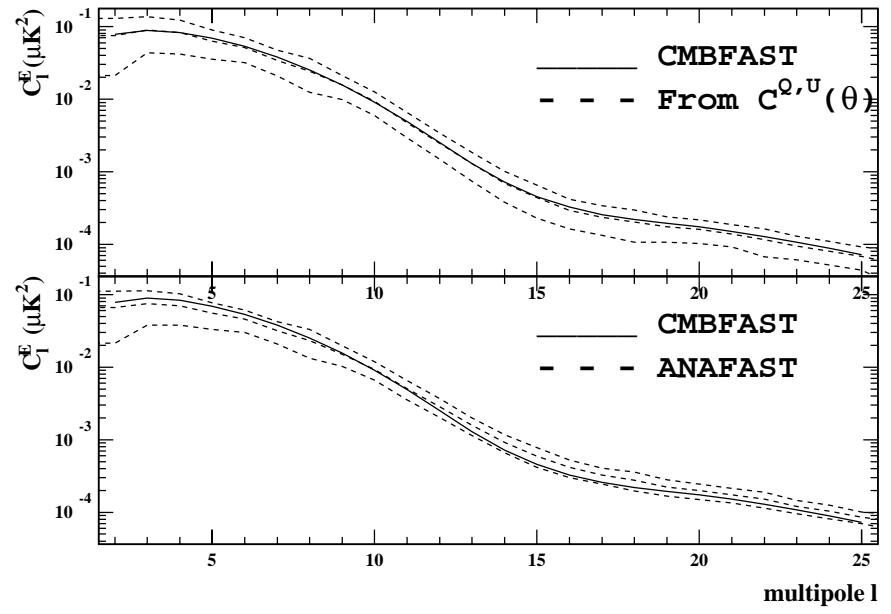

Fig. 2. $C_{\ell}^{\mathrm{E}}$ power spectrum of our selected CMB model, convolved with the SPOrt nominal $F W H M$ of $7^{\circ}$ (solid), compared to the average of 100 power spectra measured by either our method (top) or ANAFAST (bottom) on simulated maps featuring the SPOrt cuts. Measured 1- $\sigma$ bands due to cosmic variance are shown as well.

$\Omega_{\Lambda}=0.7, \tau=0.2, H_{0}=65 \mathrm{~km} \mathrm{~s}^{-1} \mathrm{Mpc}^{-1}, \Omega_{\mathrm{b}}=0.05$ and no contributions from gravitational waves. For the synchrotron radiation we use $Q$ and $U$ map templates at $22 \mathrm{GHz}$ developed by Bernardi et al. (2003) and featuring a $P_{\text {rms }}=\sqrt{\left\langle Q^{2}+U^{2}\right\rangle} \simeq$ $35 \mu \mathrm{K}$. The polarized intensity peak emission is about $130 \mu \mathrm{K}$. The main ingredients of the template are total intensity low frequency data (Haslam et al. 1982; Reich 1982), from which the syncrothron polarized intensity is extracted, and optical starlight data (Heiles 2000), from which polarization angles are derived. The template frequency has been chosen to correspond to the SPOrt channel where synchrotron emission is expected to be most important.

Noise streams to be added to the sky signal are built in the frequency space, where their Fourier coefficients are generated according to the spectrum defined in Eq. (1), with $\beta=1$, and transported to the time domain via FFT. Our code allows us to calculate the angle between our polarimeters and the longitudelatitude fixed reference frame for each data sample, and to set the sampling frequency from the nominal rate $(1 \mathrm{~Hz})$ to any other rate while keeping the SPOrt nominal istantaneous sensitivity of $1 \mathrm{mK} \mathrm{s}^{1 / 2}$ (Carretti et al. 2003). In the tests described here the sampling rate was set to $1 \mathrm{~Hz}$, and the SPOrt observing time was about 1 year. The final output of our simulations are TOD and noise arrays, clean $Q$ and $U$ signal maps to investigate the effects of SPOrt partial sky survey, $Q$ and $U$ maps containing both signal and noise before and after destriping as well as a hit map to evaluate the time spent over each map pixel. We use the HEALPix ${ }^{5}$ pixelisation scheme (Gorski et al. 1998) with $N$ side $=64$ to ensure the effects of pixelisation are negligible when compared to the antenna beam (the pixel size of the output maps is $\simeq 1^{\circ}$ ). We test the destriping efficiency on simulated maps by evaluating the residual low frequency noise in terms of added rms noise with respect to the white noise case, angular correlation functions $C^{X}(\theta)(X=Q, U)$ and power spectra $C_{\ell}^{Y}$ $(Y=E, B)$.

\footnotetext{
${ }^{5}$ http://www.eso.org/science/healpix
}

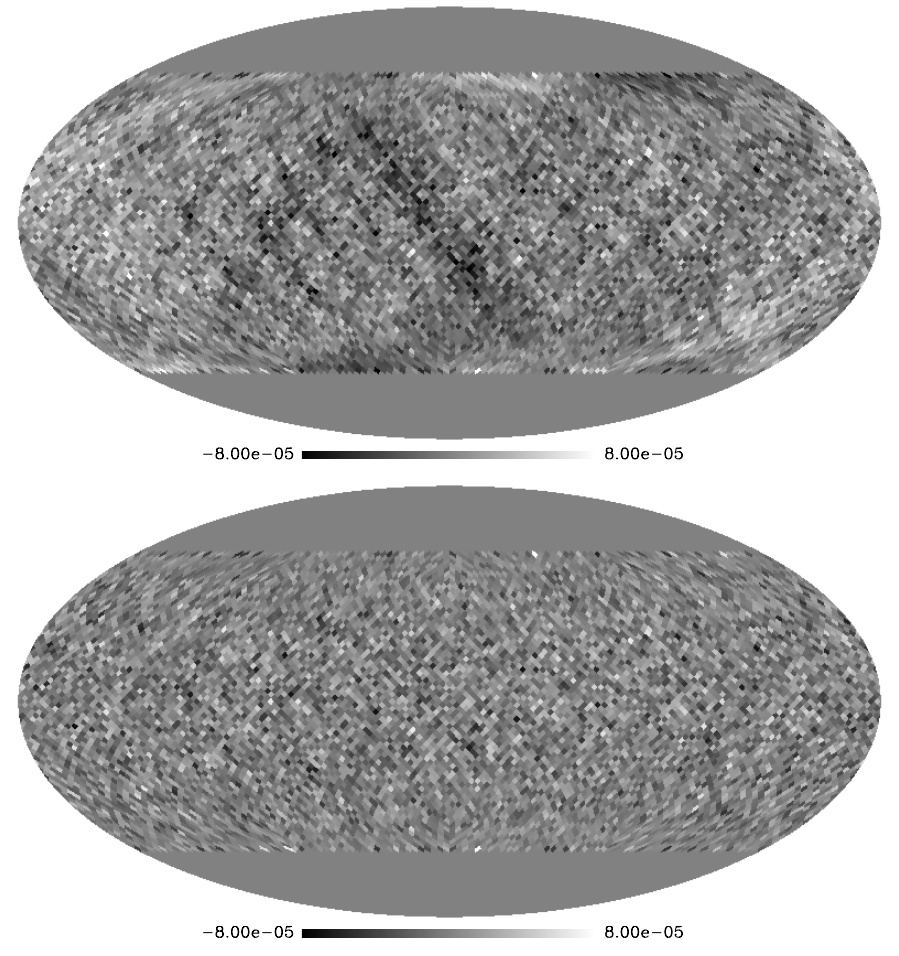

Fig. 3. Noise simulated maps before (top) and after (bottom) destriping, for the case $f_{\mathrm{k}}=1.8 \times 10^{-4} \mathrm{~Hz}$, in Kelvin. The HEALPix parameter $N$ side is 32 .

\subsection{Correlation functions and power spectra}

To avoid edge effects due to partial sky coverage and to efficiently take into account the presence of noise, even in case of non uniform sky coverage, we primarly test our destriping technique by studying the two-point correlation functions $C^{X}(\theta)$ $(X=Q, U)$ measured from our simulated maps. We sample the correlation functions with about one degree of angular resolution according to the expression:

$\tilde{C}^{X}(\theta)=\frac{\sum_{i j} w_{i j} \Delta_{i}^{X} \Delta_{j}^{X}}{\sum_{i j} w_{i j}}$

Here $\Delta_{i}^{X}$ is the content of pixel $i$ of map $X$, and $w_{i j}$ is the weight of the $i j$ pixel pair

$w_{i j}=\frac{2 w_{i} w_{j}}{w_{i}+w_{j}}$

where each pixel weight $w_{i}$ is proportional to the pixel observing time and $\sum_{i=1}^{N_{\mathrm{pix}}} w_{i}=N_{\mathrm{pix}}$. In the computation the Stokes parameters are properly rotated so that $Q$ is defined along the great circle connecting the points in each pair (Zaldarriaga 1998). The exact value of the angle $\theta$ at which the correlation functions are sampled is calculated as the weighted average of the angles between pixels contributing to the same measured point. As an example, the measured two-point correlation functions of $Q, U$ maps containing only white noise are on average zero everywhere but at 0 -distance, where they are checked to be equivalent to the average pixel variance. 

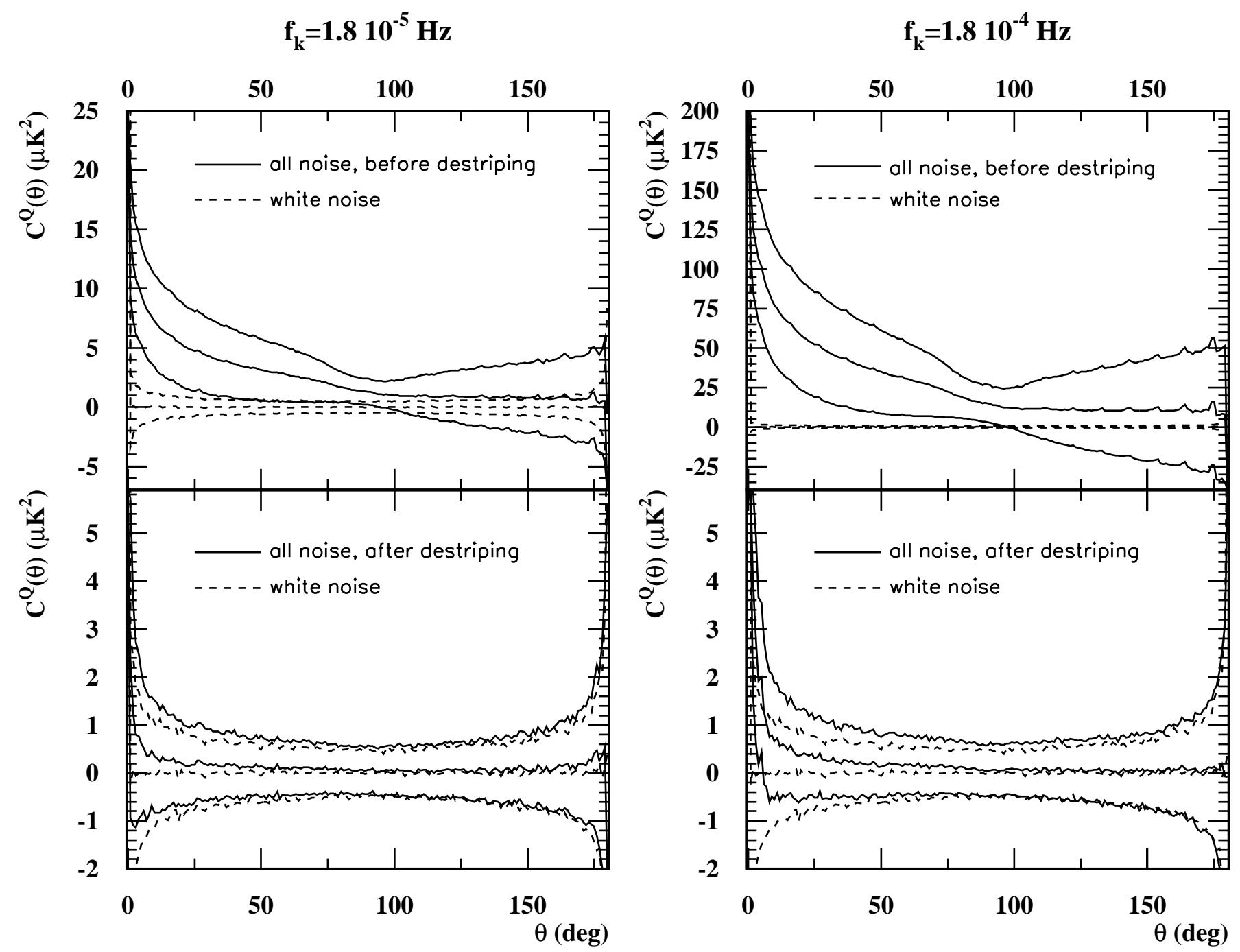

Fig. 4. Average and $1 \sigma$ band of correlation functions $C^{Q}(\theta)$ measured from 500 noise maps, before and after destriping, for two values of the knee frequency $f_{\mathrm{k}}$. Before destriping the $y$ scale is not the same for the two $f_{\mathrm{k}}$ values. Each simulation corresponds to about one year of SPOrt realistic data taking. The case of purely white noise is shown for comparison.

The $C_{\ell}^{E, B}$ power spectra can be recovered from the measured correlation functions by integration (Kamionkowski et al. 1997; Zaldarriaga 1998):

$C_{\ell}^{E}=\int_{0}^{\pi}\left[C^{Q}(\theta) F_{\ell, 2}^{1}(\theta)+C^{U}(\theta) F_{\ell, 2}^{2}(\theta)\right] \sin \theta \mathrm{d} \theta$

$C_{\ell}^{B}=\int_{0}^{\pi}\left[C^{U}(\theta) F_{\ell, 2}^{1}(\theta)+C^{Q}(\theta) F_{\ell, 2}^{2}(\theta)\right] \sin \theta \mathrm{d} \theta$

where the functions $F_{\ell, 2}^{i}(\theta)$ have been defined by Zaldarriaga (1998). We perform the integration by Gauss quadrature, where the correlation function values at the needed points $\theta$ are obtained by interpolation, with a second order polynomial, of the three closest measured points. This method of measuring power spectra is similar to that used by Szapudi et al. (2001), though here it has been extended to polarization data. It has been tested on full-sky maps of simulated CMB emission, convolved with a Gaussian filter with $F W H M=7^{\circ}$, by comparing the measured power spectra to those obtained by using the ANAFAST package: we get the same results, within numerical precision, up to $\ell=25$, i.e. in the full multipole range covered by SPOrt. On partial sky maps this method is found to work better than ANAFAST, thanks to the lack of edge effects: our average of power spectra extracted from CMB maps without noise and featuring the SPOrt sky coverage matches the input theoretical spectrum very well, whereas that measured with ANAFAST is flatter, as shown in Fig. 2. Furthermore, when trying to extract the real sky signal from noisy maps this method allows for pixel weighting in case of non-uniform sky coverage as well as noise subtraction, provided the noise statistical properties (correlation functions) are known. It has the additional advantages of being simple and rather fast: the required computing time practically corresponds to that needed to calculate the twopoint correlation functions, i.e. about $35 \mathrm{~min}$ on our platform. The number of operations is $O\left(N_{\text {pix }}^{2}\right)$, while optimal methods to measure power spectra require matrix inversions tipically implying a number of operations $O\left(N_{\text {pix }}^{3}\right)$ (Wright 1996). The implementation of a new algorithm for computing $N$-point correlation functions is expected to further reduce the computing time (Moore et al. 2001). 


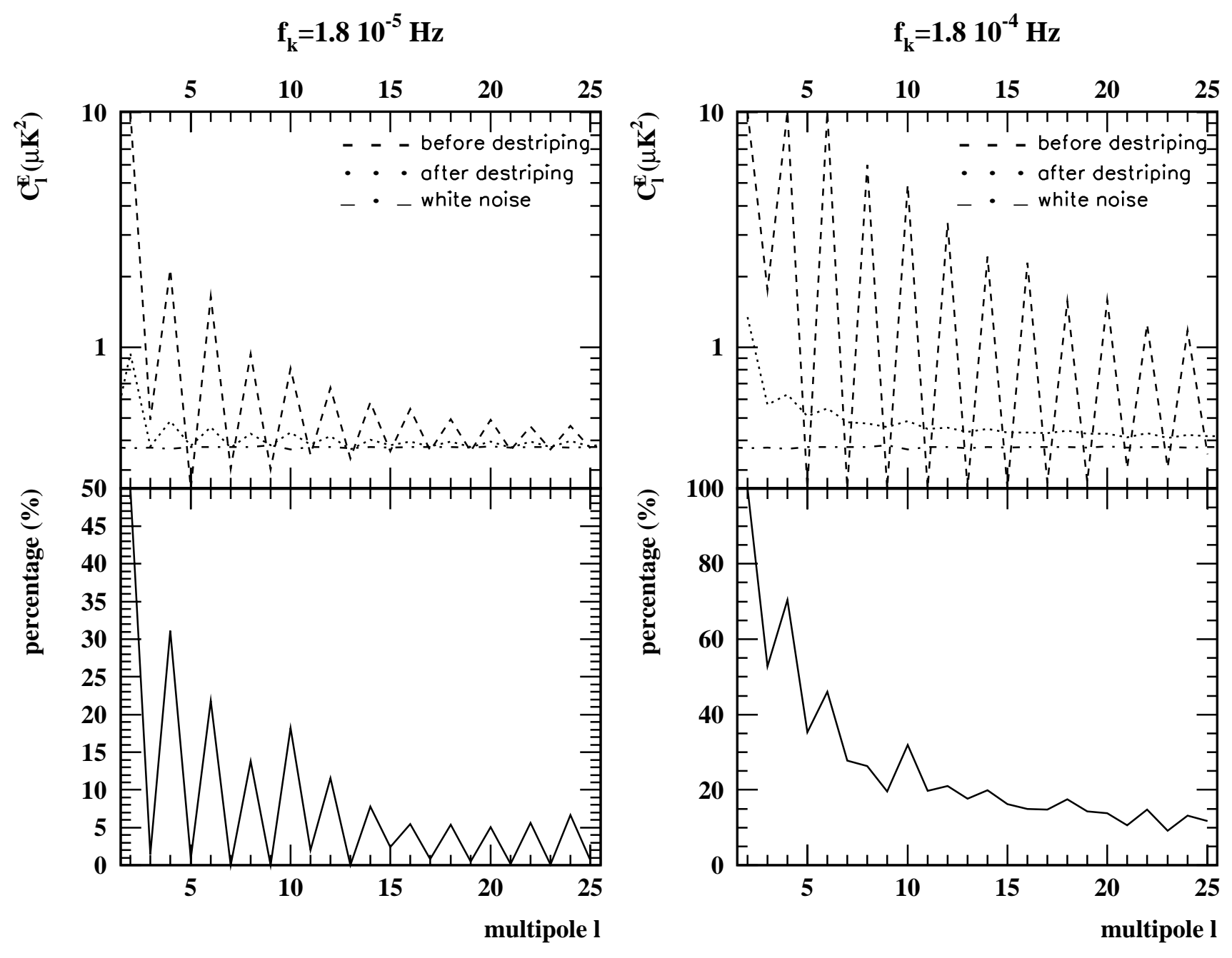

Fig. 5. Average of noise power spectra measured from the same maps used to make Fig. 4 (top). In the bottom panel the residual noise in percentage of the measured white noise level is shown as a function of the multipole.

\subsection{Destriping signal-only maps}

To check for the presence of spurious correlations introduced by our algorithm and depending on the underlying signal, as a first test we run the destriping code over CMB and Synchrotron maps containing no noise. The test confirms our algorithm subtracts the average signal from the input map when dealing with scalar quantities (CMB temperature in our case), whereas nothing is lost in our $Q$ and $U$ maps. We then analyse the difference between the destriped and the original maps as a function of the number of iterations of the destriping cycle. Should no residual effects be present, these differences would asimptotically become null for $Q$ and $U$ maps, and a constant number corresponding to the average of the input signal for scalar maps. Indeed, the maximum pixel-to-pixel difference, which depends on the level of the underlying signal, decreases with increasing number of iterations. In particular, the spurious noise introduced when destriping maps of synchrotron emission at $22 \mathrm{GHz}$, i.e. the SPOrt frequency where the Galactic contribution is expected to be higher, becomes negligible also for CMBP after about 20 loops, reaching the tiny level of few $\mathrm{nK}$ after 30 loops. More details are shown in Table 1, both in
Table 1. Peak-to-peak amplitude and rms of the spurious noise introduced when destriping signal-only maps (CMB and synchrotron), as functions of the iteration loop number, for $Q$ and $U$ maps. The last line shows the peak-to-peak amplitude and rms of the underlying $Q \& U$ signals.

\begin{tabular}{lllll}
\hline \hline$N_{\text {loops }}$ & \multicolumn{3}{c}{ cmb } & \multicolumn{2}{c}{ sync } \\
\hline & $\begin{array}{l}\text { peak-to-peak } \\
\text { amplitude }[\mu \mathrm{K}]\end{array}$ & rms & $\begin{array}{l}\text { peak-to-peak } \\
\text { amplitude }[\mu \mathrm{K}]\end{array}$ & $\mathrm{rms}$ \\
& {$[\mu \mathrm{K}]$} & \\
\hline 10 & $3 \times 10^{-2}$ & $4 \times 10^{-3}$ & 1.1 & 0.3 \\
20 & $2 \times 10^{-3}$ & $4 \times 10^{-4}$ & $8 \times 10^{-2}$ & $2 \times 10^{-2}$ \\
30 & $3 \times 10^{-4}$ & $3 \times 10^{-5}$ & $1 \times 10^{-2}$ & $2 \times 10^{-3}$ \\
40 & $1 \times 10^{-4}$ & $4 \times 10^{-6}$ & $5 \times 10^{-3}$ & $2 \times 10^{-4}$ \\
\hline & 3 & 0.5 & 170 & 20 \\
\hline
\end{tabular}

terms of peak-to-peak amplitude and rms of spurious noise. For comparison, the peak-to-peak amplitude and rms of the underlying signal maps are shown as well.

The same conclusions can be derived by inspecting the angular correlation functions $C^{X}(\theta)(X=Q, U)$ of the spurious 
Table 2. Excess rms noise due to low-frequency contributions, with respect to the white noise level, for pixels of $\simeq 7^{\circ}$.

\begin{tabular}{lll}
\hline \hline$f_{\mathrm{k}}(\mathbf{H z})$ & Before Destriping & After Destriping \\
\hline $1.8 \times 10^{-4}$ & $310 \%$ & $6 \%$ \\
$1.8 \times 10^{-5}$ & $35 \%$ & $<1 \%$ \\
\hline
\end{tabular}

noise: they are not flat, but deviations from a flat null function decrease with increasing number of iteration cycles and can be made negligible after about 10 (20) loops when destriping CMB (synchrotron) $Q, U$ maps.

\subsection{Destriping noise maps}

The effectiveness of our destriping technique is then tested on simulated maps containing only noise, in the following cases:

- white noise,

- $1 / f$ noise,

- both white and $1 / f$ noise.

The good performances of the technique are made evident in Fig. 3 where we show a simulated noise map (both $1 / f$ and white) before and after destriping.

One possible way to quantify the residual correlated noise after destriping is measuring the fractional excess pixel noise with respect to the case of purely white noise. Results are shown in Table 2 for two different values of the knee frequency, corresponding to the SPOrt goal knee frequency, $f_{\mathrm{k}}=$ $1.8 \times 10^{-5} \mathrm{~Hz}$, and the SPOrt orbit frequency, $f_{\mathrm{o}}=1.8 \times 10^{-4} \mathrm{~Hz}$, the latter representing a very conservative case.

Another way to quantify the residual correlated noise is measuring and inspecting the two-point correlation functions $C^{X}(\theta)$ of simulated $Q$ and $U$ noise maps. Averages and $1 \sigma$ bands of 500 correlation functions $C^{Q}(\theta)$ measured from maps containing both white and $1 / f$ noise, before and after destriping, are compared to the purely white noise case in Fig. 4 for the same knee frequencies as in the previous test. The correlation functions $C^{U}(\theta)$ are similar and are not shown. As expected, the correlated noise is strongly reduced by the destriping procedure, the residuals falling within the statistical error of the white noise case for $f_{\mathrm{k}}=f_{\mathrm{k}}^{\text {goal }}$. The correlation functions for the case of purely $1 / f$ noise are similar to the white plus $1 / f$ case everywhere but at 0 distance, and are not shown.

If the noise statistical properties are known, its twopoint correlation function after destriping can be calculated with Monte Carlo techniques and subtracted from the measured values before integrating, the recovering of signal power spectra presenting no huge problems. However, the residual correlated noise also increases the expected error on measured quantities. In our case the rms of correlation functions measured from many noise maps is increased by roughly $4 \%(11 \%)$, for $f_{\mathrm{k}}=1.8 \times 10^{-5} \mathrm{~Hz}\left(f_{\mathrm{k}}=1.8 \times 10^{-4} \mathrm{~Hz}\right)$, with respect to the case of purely white noise.

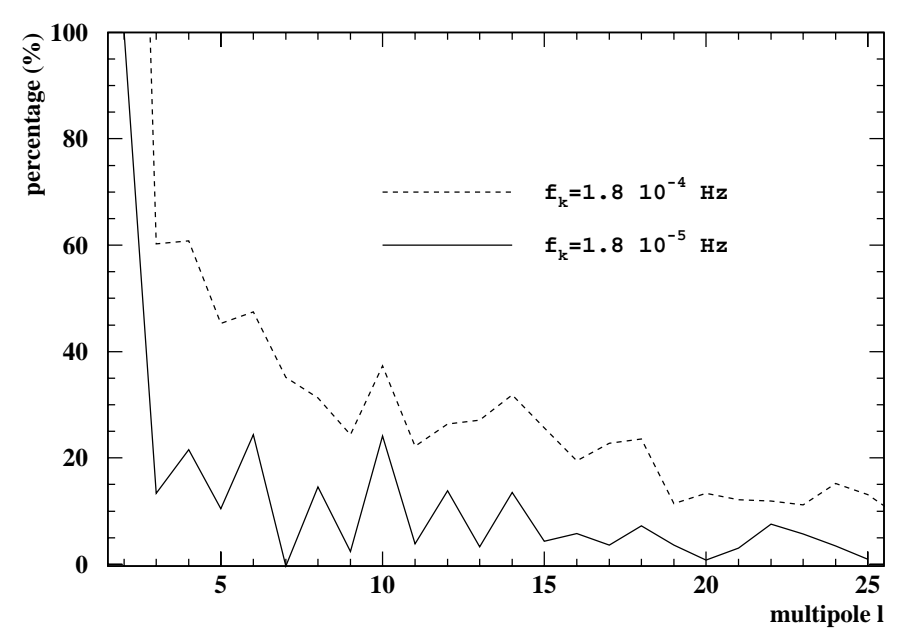

Fig. 6. Increment, due to the presence of residual correlated noise, in the rms of measured noise power spectra, after destriping, as a function of the multipole, in percentage of the rms of purely white-noise power spectra.

For completeness, and to ease the comparison with other methods, average noise power spectra, obtained from the correlation functions used to make Fig. 4, are shown in Fig. 5, though they carry the same information as the correlation functions. As expected, even for the largest knee frequency the $C_{\ell}^{E}$ power spectrum of the residual noise is close to that of purely white noise. The region of low multipoles is the most sensitive to low frequency residuals, some contributions being always found here also after the application of other destriping techniques (Maino et al. 1999). The excess noise, in percentage of the measured white noise level, is shown in the bottom panel of Fig. 5 as a function of the multipole. The theoretical white noise level can be calculated as follows:

$C_{\ell}^{\mathrm{wn}}=\frac{4 \pi}{N_{\mathrm{pix}}}\left\langle\sigma^{2}\right\rangle$

where $\left\langle\sigma^{2}\right\rangle$ represents the average noise variance per pixel. Our measured value is equivalent to its expectation of $0.37 \mu \mathrm{K}^{2}$, again confirming that our map-making algorithm is correct.

Finally, Fig. 6 shows the increment, due to the presence of residual correlated noise, in the rms of measured noise power spectra, after destriping, in percentage of the rms of purely white noise power spectra, again as a function of the multipole.

\section{Conclusions}

We presented a new iterative destriping technique working directely on $Q$ and $U$ Stokes parameter data as well as on scalar quantities. As for other methods, in order for the technique to work properly the receiver knee frequency must be lower than the experiment modulation frequency, and there must be sufficient overlap between different orbits. On the other hand, no requirements on the statistical properties of the noise are necessary here. Moreover, our observing strategy preserves average $Q$ and $U$ values in the destriped maps, though the map average is still lost when dealing with scalar quantities. 
The performances of the technique, studied on simulated SPOrt data by analysing both the measured $C^{Q, U}(\theta)$ two-point correlation functions and the measured $C_{\ell}^{E, B}$ power spectra, are comparable to those of other methods based on $\chi^{2}$ minimisation. Computer time is not an issue, no matrix inversions being involved.

Power spectra are recovered from the measured two-point correlation functions by integration, this method being implemented here for the first time for polarization data. It has the advantages of avoiding edge problems arising when using the ANAFAST code and to allow for pixel weighting in case of non uniform sky coverage.

Acknowledgements. This work has been carried out in the context of the SPOrt program, which is funded by the Italian Space Agency. We thank B. Audone and F. Amisano for useful discussions and the anonimous referee for good suggestions. We acknowledge the use of CMBFAST and HEALPix packages.

\section{References}

Berkhuijsen, E. M. 1975, A\&A, 40, 311

Bernardi, G., Carretti, E., Fabbri, R., et al. 2003, MNRAS, submitted Bruscoli, M., Tucci, M., Natale, V., et al. 2002, New Astron., 7, 171

Carretti, E., Tascone, R., Cortiglioni, S., Monari, J., \& Orsini, M. 2001, New Astron., 6, 173

Carretti, E., Cortiglioni, S., Bernardi, G., et al. 2003, in Polarimetry in Astronomy, SPIE Symposium on Astronomical Telescopes and Instrumentation, Waikoloa, Hawaii, August 20-28 2002, SPIE Conf. Proc. 4843, in press [astro-ph/0212067]

Cortiglioni, S., Baralis, M., Bernardi, G., et al. 2002, in AMiBA 2001, ed. L.-W. Chen, C.-P. Ma, K.-W. Ng, \& U.-L. Pen, Taipei \& Hualien, June 11-15 2001, ASP Conf. Ser., CS-257, 243

Delabrouille, J. 1998, A\&AS, 127, 555
Górski, K. M., Hivon, E., \& Wandelt, B. D. 1998, in MPA/ESO Conf. on Evolution of Large Scale Structures: from recombination to Garching, ed. A. J. Banday, R. K. Shet, \& L. Da Costa, http://www.mpa-garching.mpg.de/english/conferences/ elss98/contributions 1998.html [astro-ph/9812350]

Haslam, C. G. T., Stoffel, H., Salter, C. J., \& Wilson, W. E. 1982, A\&AS, 47, 1

Heiles, C. 2000, AJ, 119, 923

Janssen, M. A., Scott, D., White, M., et al. 1996, Internal Report PSI-96-01 [astro-ph/9602009]

Kamionkowski, M., Kosowsky, A., \& Stebbins, A. 1997, Phys. Rev. D, 55,7368

Kamionkowski, M., \& Kosowsky, A. 1998, Phys. Rev. D, 57, 685

Lazarian, A., \& Prunet, S. 2002, in Astrophysical Polarized Backgrounds, ed. S. Cecchini, S. Cortiglioni, R. Sault, \& C. Sbarra, AIP Conf. Proc., 609, 32

Maino, D., Burigana, C., Maltoni, M., et al. 1999, A\&AS, 140, 383

Moore, A. W., Connolly, A., Genovese, C., et al. 2001, in Mining the Sky, Garching, Germany, 31 July-4 August, 2000, ed. A. J. Banday, S. Zaroubi, \& M. Bartelmann (Heidelberg: SpringerVerlag), 71 [astro-ph/0012333]

Reich, W. 1982, A\&AS, 48, 219

Revenu, B., Kim, A., Ansari, R., et al. 2000, A\&AS, 142, 499

Seljak, U. 1997, ApJ, 482, 6

Szapudi, I., Prunet, S., Pogosyan, D., Szalay, A. S., \& Bond 2001, ApJ, 548, L115

Tegmark, M. 1997a, ApJ, 480, L87

Tegmark, M. 1997b, Phys. Rev. D, 56, 4514

Tegmark, M., Eisenstein, D. J., Hu, W., \& de Oliveira-Costa, A. 2000, ApJ, 530, 133

Wollack, E. J. 1995, Rev. Sci. Instrum., 66, 4305

Wollack, E. J., \& Pospieszalski, M. W. 1998, IEEE MTT-S Digest, 669

Wright, E. L. 1996, in IAS CMB Data Analysis Workshop [astro-ph/9612006]

Zaldarriaga, M. 1998, Ph.D. Thesis, M.I.T. [astro-ph/9806122]

Zaldarriaga, M., Spergel, D. N., \& Seljak, U. 1997, ApJ, 488, 1 